\title{
STRUCTURE OF THE LOCAL GALACTIC MAGNETIC FIELD
}

\author{
R. J. Rand and S. R. Kulkarni \\ California Institute of Technology, 105-24 \\ Pasadena, CA 91125
}

\begin{abstract}
We have modeled the local Galactic magnetic field using pulsar rotation measures (RMs), of which there are now about 200 available. The North Polar Spur has a significant effect on pulsar RMs. Using RMs of 116 pulsars nearer than $3 \mathrm{kpc}$, we find that the local field has a strength $B_{0}=1.6 \pm 0.2 \mu G$ toward longitude $l_{B}=96^{\circ} \pm 4^{\circ}$, with a reversal of the field at a distance $D_{r}=600 \pm 80 \mathrm{pc}$ toward the inner Galaxy. Relaxing the $3 \mathrm{kpc}$ distance restriction, we find that a concentric ring model with reversals is superior to a bisymmetric spiral model as a fit to the data.
\end{abstract}

\section{Introduction}

There have been many attempts in the last 15 years to model the Galactic magnetic field using RMs of pulsars and extragalactic sources (Manchester 1974, hereafter M74; Thomson and Nelson 1980, hereafter TN; Simard-Normandin and Kronberg 1980, hereafter SK; Sofue and Fujimoto 1983, hereafter SF; Inoue and Tabara 1980, hereafter IT), resulting in much disagreement over whether or not the field is locally aligned with the spiral arms, and whether globally it follows the spiral pattern of the Galaxy or has a ringlike geometry. SK and SF have found good evidence for reversals of the large-scale field direction.

Recently, Hamilton and Lyne (1987) determined RMs for 163 pulsars, thus greatly increasing the data available for analysis of the Galactic magnetic field. We have used the large body of pulsar RM data and used them in a new analysis of the Galactic field. The results presented here are described in greater detail in a recent paper (Rand and Kulkarni 1989; hereafter RK). Our study of the field irregularities is not discussed here, but is described at length in RK. There we conclude that simple "single-cell-size" models of the random field fail to provide an adequate description of the irregularities. We conclude instead that the local anomalous magnetic features - which show ordered fields on a variety of length-scales, are probably prominent throughout the interstellar medium, and explain at least in part the complexity of the irregular component.

\section{Selection of the Data Set}

It is important to identify local features of the sky, such as nearby supernova remnants, which cause anomalous RMs over regions of large angular extent. Pulsars lying behind such regions should not be included in any modeling, since their RMs will systematically bias 
the best-fit parameters. Figure 1 shows derived pulsar $B_{\|}$'s $\left(B_{\|}=1.232 R M / D M \mu G\right)$ in Galactic longitude and latitude coordinates. Several familiar trends appear in this figure. The negative $B_{\|}$'s tend to cluster in the first two quadrants, while the positive ones are generally found in quadrants III and IV, and in the region $0^{\circ} \lesssim l \lesssim 60^{\circ}, b>0^{\circ}$.

The North Polar Spur (NPS), which is a nearby $(D \sim 100 \mathrm{pc})$ continuum feature of large angular extent located at $l \approx 30^{\circ}, 0^{\circ} \lesssim b \lesssim 25^{\circ}$, has often been pointed out as a possible source of anomalous RM (SK, IT, M74). Figure 1 shows, as have previous studies, that there is a clear deviation from the large-scale symmetry in $l$ and $b$ in the region $0^{\circ}<l<60^{\circ}$, $b>0^{\circ}$. We believe this deviation is due to these nearby features. We have therefore not included pulsars with $\left|B_{\|}\right|>2 \mu G$ in our modeling. Two other regions, Region A (so named by SK), at $60^{\circ}<l<140^{\circ},-40^{\circ}<b<10^{\circ}$, and the Gum Nebula, centered at $l=255^{\circ}, b=0^{\circ}$, are not fould to have a significant enough effect on observed $B_{\|}$'s to warrant flagging out any pulsars (see RK for more discussion of these regions).

\section{Results for the Large-Scale Field}

Our first model, for nearby pulsars only, assumes that the local field is linear, with an unknown orientation and a possible reversal of its direction toward the inner Galaxy, occurring at a distance $D_{r}$, measured along a line perpendicular to the direction of the field. We first performed a $\chi^{2}$ analysis using all 130 pulsars within $D=3 \mathrm{kpc}$, regardless of whether they had been flagged for lying behind the NPS. We obtained $B_{0}=1.4 \pm 0.3 \mu G, l_{B}=77^{\circ} \pm 3^{\circ}$, and $D_{r}=180 \pm 60 \mathrm{pc}$. The results for $l_{B}$ and $D_{r}$ are in good agreement with TN, who did not flag any pulsars in their sample of 48 , with the reasoning that "these sources simply exhibit local aspects of the random magnetic field component." But in fact, as shown in the present analysis, the "NPS pulsars" have biased the best-fit values of $l_{B}$ and $D_{r}$, because the NPS has a systematic effect on RMs, not a random one.

Throwing out the "NPS pulsars," we repeated the analysis on the remaining 116 pulsars and obtained $B_{0}=1.6 \pm 0.2 \mu G, l_{B}=96^{\circ} \pm 4^{\circ}$, and $D_{r}=600 \pm 80 \mathrm{pc}$. Our result for $B_{0}$ agrees with that of IT, but is much lower than TN's value of $B_{0}=3.5 \mu G$. Such a high value was probably found because of the extremely small scale height $(75 \pm 40 \mathrm{pc})$ they determined for the field. This scale height is more than an order of magnitude smaller than values derived by IT and SK, and, as pointed out by IT, is smaller than that of the electrons by about the same factor. We agree with IT that the pulsar RM data used by TN did not justify a five parameter model, and that their results are suspect. The result of $l_{B}=96^{\circ}$ obtained with the restricted data set is in good agreement with M74's and IT's results.

For our bisymmetric spiral and concentric ring models, the $D \lesssim 3 \mathrm{kpc}$ restriction was relaxed. In summary, we found the former model is inappropriate since it predicts a spiral pattern with a pitch angle in disagreement with known spiral tracers. We therefore prefer the concentric ring model for the field geometry. This model includes periodic reversals with radius of the field direction. See RK for a full discussion.

\section{Conclusions}

We have analyzed the Galactic magnetic field based on the large amount of pulsar RM data now available. The North Polar Spur appears to cause large positive RMs in the region $0^{\circ}<l<60^{\circ}, 0^{\circ}<b<60^{\circ}$, and thus causes a systematic bias in modeling if the pulsars in this region are not flagged. Using only unflagged pulsars within $D=3 \mathrm{kpc}$, and 
a longitudinal model of the local field, we find that the strength is $B_{0}=1.6 \pm 0.3 \mu G$ in the direction $l_{B}=96^{\circ} \pm 4^{\circ}$, with a reversal at $D_{r}=600 \pm 80 \mathrm{pc}$ toward the inner Galaxy. A bisymmetric spiral model of the Galactic field is inappropriate since it predicts a spiral pattern with a pitch angle in disagreement with known spiral tracers. We therefore prefer a concentric ring geometry for the field of our Galaxy.

Vallée (1984), in a study of local magnetic bubbles, came to essentially the same conclusion about the geometry of the local field - that if proper account is made of local anomalous magnetic features, the field appears to be ringlike instead of parallel to the spiral arms. There is now convincing evidence from several studies (see RK) that the magnetic field of our Galaxy has a circular geometry.

\section{References}

Hamilton, P. A. and Lyne, A. G. 1987, M. N. R. A. S. 224, 1073.

Inoue, M. and Tabara, H. 1981, Publ. Astr. Soc. Jpn 33, 603 (IT).

Manchester, R. N. 1974, Ap. J. 188, 637 (M74).

Rand, R. J. and Kulkarni, S. R. 1989, Ap. J. 343, 760.

Simard-Normandin, M., and Kronberg, P. P. 1980, Ap. J. 242, 74 (SK).

Sofue, Y. and Fujimoto, M. 1983, Ap. J. 265, 722 (SF).

Thomson, R. C. and Nelson, A. H. 1980, M. N. R. A. S. 191, 863 (TN).

Vallée, J. P. 1984, Astr. Ap. 136, 373.
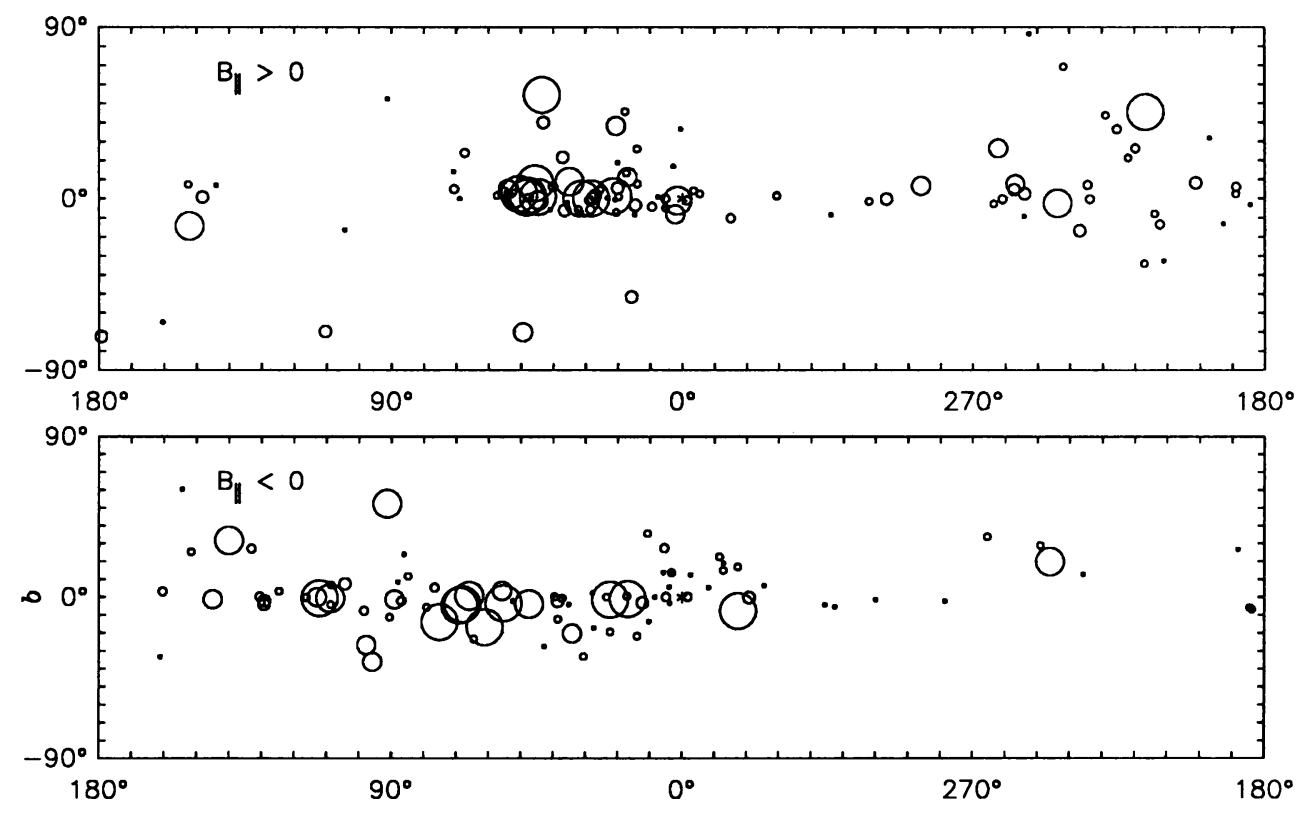

Figure 1. View of $B_{\|}$'s in Galactic coordinates for pulsars with $D<3 \mathrm{kpc}$. (a) positive $B_{\|}$'s and $(b)$ negative $B_{\|}$'s. The size of the plotting symbol represents the magnitude of $B_{\|}$in steps of $0.5 \mu G$, with the largest symbol representing $\left|B_{\|}\right|>3.0 \mu G$, and the smallest symbol representing $\left|B_{\|}\right|<0.5 \mu G$. 